\title{
Jets from subpc to kpc scale
}

\author{
Fabrizio Tavecchio \\ Osservatorio Astronomico di Brera-Merate, Via Bianchi 46, Merate-Italy
}

\begin{abstract}
The Chandra discovery of bright X-ray emission from kpc-scale jets provides us unprecedented insights into the physical state of the plasma in the flow. In particular it is possible to get good constraints on the power and pressure in bright knots. For a group of selected sources with blazar-type cores it is also possible to constrain the physical quantities of the jet at sub-pc scale. We discuss how these results can help us to connect the properties of the jet at different scales.
\end{abstract}

Key words: Galaxies: jets; Quasar: general; X-rays: galaxies

\section{Introduction}

The study of extragalactic jets has been recently renewed by the discovery, made by Chandra, of numerous jets bright in the X-rays at scales $>\mathrm{kpc}$, both in FRI and FRII sources (Chartas et al. 2000, Sambruna et al. 2002). While in low-power FRI jets the dominating emission mechanism responsible for X-rays is thought to be synchrotron from very-high energy electrons (e.g. Worrall et al. 2001), in the case of the most powerful sources it is clear that $\mathrm{X}$-rays constitute a second spectral component, likely due to the IC scattering of the CMB photons (Tavecchio et al. 2000a, Celotti et al. 2001), implying that X-ray bright jets are still relativistic (with bulk Lorentz factors $5-10$ ) at $\sim 100 \mathrm{kpc}$.

At the opposite side of scalelengths, studies of the innermost portion $(d \sim$ $0.1 \mathrm{pc}$ ) of jets in AGNs are rather well developed. Our knowledge of these regions of jets is mainly based on blazars, whose Spectral Energy Distribution (extending from radio to $\gamma$-rays) is dominated by the relativistic amplified nonthermal continuum produced in a jet closely aligned to the line of sight (e.g. Urry \& Padovani 1995). The double humped SED of these sources is well described by synchrotron-IC models (e.g., Ghisellini et al. 1998; Sikora \& Madejski 2001).

The possibility to constrain the physical state of the plasma in the jet both at 
sub-pc and kpc scale offers us the interesting opportunity to shed some light on the evolution of the jet from very small scales, close to the central engine, to the outer regions, where the jet is starting to significantly decelerate. In this work we present a first step, reporting the results obtained for a small group of sources for which good data for both regions are available. These sources are PKS 1510-089, 1641+399, PKS 0521-365 and 3C371. The emission models from the blazar region and the parameters for the first three sources are discussed in Tavecchio et al. (2000b, 2002), while the model for 3C371 is discussed in Tavecchio et al. (in prep). Multiwavelength (Chandra, HST and radio) data for extended jets are discussed in Gambill et al. (in prep 1510-089; 1641+399), Pesce et al. (2001, 3C371) and Birkinshaw et al. (2002, 0521-365). Clearly the X-ray emission for the first two sources is well reproduced by the $\mathrm{IC} / \mathrm{CMB}$ model, while in the other two jets the dominant contribution is due to synchrotron emission. The detailed modelling of the data will be reported in Tavecchio et al. (in prep). In the following we limit the discussion to the result of the comparison of the most relevant quantities of the jet as inferred in the two regions.

\section{Results: power and pressure}

A fundamental global quantity of jets is the total (particles + magnetic field) power transported by the flow. A first result of our analysis (although strickly valid only in the case of IC/CMB sources, for which all the quantities can be univocally evaluated) is that the power derived at subpc scale is rather close to the power inferred at kpc scale. This results (for the specific case of PKS 1510-089) is presented in Fig. 1a, where we report the power estimated in the core region (horizontal dashed line) and that at the kpc-scale jet expected for different $\Gamma$ factors, assuming that the emission is produced through IC/CMB (solid line), through SSC (long-dashed line) and assuming equipartition between relativistic electrons and magnetic field (short-dashed line). The intersection between the equipartition and the IC/CMB lines marks the $\Gamma$ factor used in the spectra modelling of the kpc-scale jet emission. Note that for low values of Lorentz factors the power required by the IC/CMB model increases rapidly, due to the large number of electrons required to produce the observed X-ray flux in presence of a rapidly decreasing amount of beamed CMB photons. Note also that an SSC origin of the high energy radiation would require an extremely large value of the power (exceeding $10^{50} \mathrm{erg} / \mathrm{s}$ ), as early recognized for the specific case of PKS 0637-752 (Schwartz et al. 2000).

The equality of the jet power estimated at the two scales strongly suggests that the jet experiences very little deceleration, dissipation and entrainment, at least from the core until $\sim 100 \mathrm{kpc}$. Recent numerical simulations seems to confirm these conclusions (Scheck et al. 2002), at least for distances $<100$ 

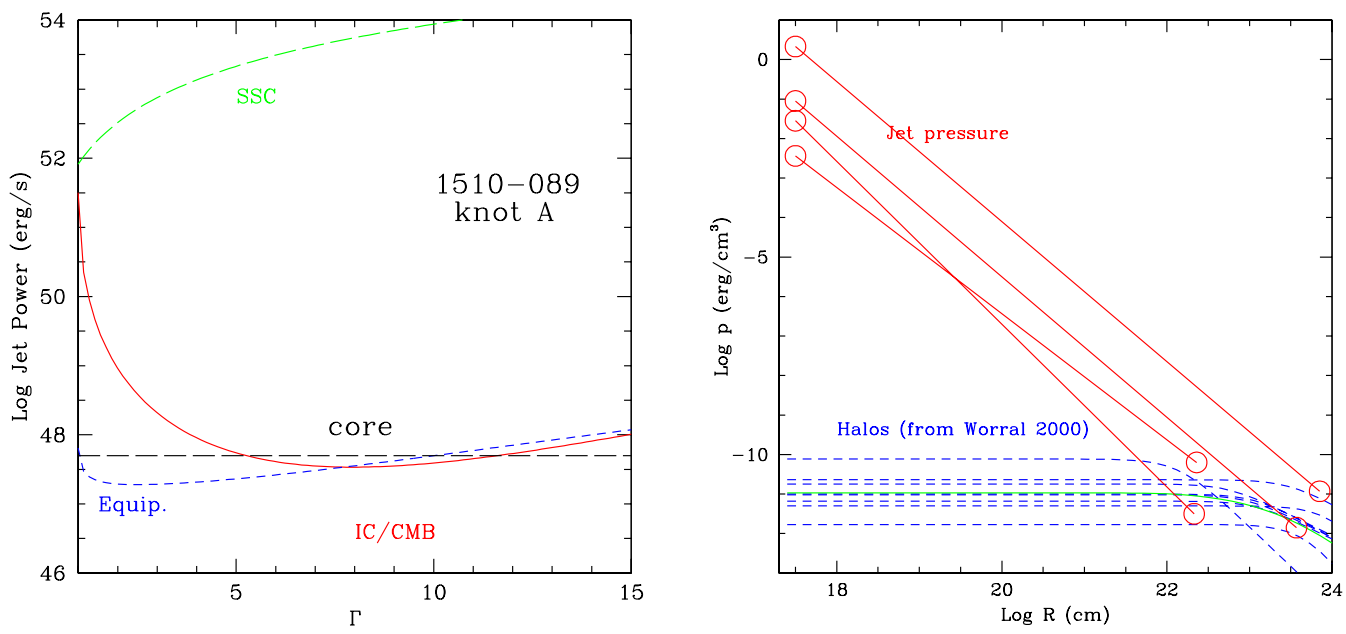

Fig. 1. Left: Jet power inferred at kpc scale and in the core for 1510-089. The power estimated using equipartition (short-dashed line) is equal to that estimated through the IC/CMB (solid line) at $\Gamma \sim 7$ and it is very close to the core power. The power required by the SSC model (long dashed) is much larger than the power usually estimated in these sources. Right: Pressure profiles for the jet discussed in the text (solid lines) and for the hot halos around a group of radio galaxies (dashed lines from Worrall \& Birkinshaw 2000). The solid line represents a logaritmic average of the profiles.

kpc. Moreover the costancy of the power implies that the central engine does not change its energy output for timescales of the order of $\sim 3 \times 10^{5}$ yrs.

In our estimates we assumed that most of the power is carried by (cold) protons (assuming the presence of 1 proton per relativistic electron). While the composition of the outer jet is not known, in the blazar region (see e.g. Tavecchio et al. 2000, Ghisellini \& Celotti 2002) the necessity of protons is clearly demonstrated by the fact that the power transported by electrons and magnetic field alone are insufficient to explain the total radiation output.

Another interesting suggestion derives considering the internal pressure of the kpc-scale knots inferred with our modelling. We recall that the estimate of the pressure is rather rubust, due to the opportunity to strongly constrain the value of the minimum Lorentz factor of the emitting electrons in the IC/CMB model (Tavecchio et al. 2000a) The derived values are in fact close to $p \sim 10^{-11}$ dyne $\mathrm{cm}^{-2}$, suggestively close to the pressure of typical hot gas halos found around FRIs (Worrall et al. 2000, Worrall et al. 2001. Recently Worrall \& Birkinshaw (2000) reported an analysis of a sample of B2 radio-galaxies with the HRI of ROSAT. Their fits of the distribution with a King profile is reported in Fig. 1b, (we also report the logaritmic average) together with our pressure estimates. Notably, the expected external gas pressure is of the same order of the pressure inferred in jets, strongly suggesting that the external gas and the jet are in pressure equilibrium. 


\section{Conclusions}

The results discussed above seems to point toward a simple scenario for the dynamical evolution of the jet from inner to the outer regions. Clearly the pressure in the blazar jet is larger than any possible confining gas. This means that, after the dissipation region, the jet expands freely until the internal pressure (gas and/or magnetic) reaches the pressure of the (supposed) hot gas of the galactic halo. At this point a reconfinement shock forms (the jet is highly supersonic), particle are accelerated, and the jet starts to decelerate (Sanders 1983, Komissarov 1994). This scenario seems to account for the typical distance at which knots form and also provide a reliable mechanism for the production of relativistic particles. A even more interesting result is that the expected pressure decay along the jet seems to be consistent with the simple profiles predicted for a free, conical expanding jet (Blandford \& Koenigl 1979). A similar scenario has been recently proposed to explain the dynamics of the jet in the FRI radio galaxy 3C31 by Laing \& Bridle (2002). The results discussed here are based on a very small group of sources: future studies along these lines could further support this scenario, providing important clues on the dynamics of relativistic jets.

\section{References}

[1] Birkinshaw, M., et al. 2002, MNRAS, 335, 142

[2] Blandford, R. D. \& Koenigl, A. 1979, ApJ, 232, 34

[3] Chartas, G. et al. 2000, ApJ, 542, 655

[4] Ghisellini, G., et al. 1998, 301, 451

[5] Ghisellini, G., \& Celotti A. 2002, ASP Conf. Proc., 258, p.273

[6] Komissarov, S. S. 1994, MNRAS, 269, 394

[7] Laing, R. A. \& Bridle, A. H. 2002, MNRAS, 336, 1161

[8] Pesce, J. E., et al. 2001, 556, L79

[9] Sambruna, R. M., et al. 2002, ApJ, 571, 206

[10] Sanders, R. H. 1983, ApJ, 266, 73

[11] Scheck, L., et al. 2002, MNRAS, 331, 615

[12] Schwartz, D. A. et al. 2000, ApJ, 540, L69

[13] Sikora, M. \& Madejski, G. 2001, High Energy Gamma-Ray Astronomy, 275

[14] Tavecchio, F., et al. 2000a, ApJ, 544, L23

[15] Tavecchio, F. et al. 2000b, ApJ, 543, 535

[16] Tavecchio, F. et al. 2002, ApJ, 575, 137

[17] Urry, C. M. \& Padovani, P. 1995, PASP, 107, 803

[18] Worrall, D. M. \& Birkinshaw, M. 2000, ApJ, 530, 719

[19] Worrall, D. M., et al. 2001, MNRAS, 326, L7 